\title{
COST 296 MIERS: conclusion
}

\author{
Alain Bourdillon ( $\left.{ }^{1}\right)$, Ljiljana R. Cander $\left({ }^{2}\right)$ and Bruno Zolesi $\left({ }^{3}\right)$ \\ ( $\left.{ }^{1}\right)$ IETR, Université de Rennes 1, France \\ ( ${ }^{2}$ STFC, Rutherford Appleton Laboratory, Chilton, UK \\ $\left(^{3}\right)$ Istituto Nazionale di Geofisica e Vulcanologia, Roma, Italy
}

A brief summary is given here of the major achievements of the COST 296 Action MIERS.

\section{Introduction}

The need for more reliable and efficient communications services, especially those involving ionospheric HF communications and navigational systems, imposes increasing demand for a better knowledge of the effects imposed by the Earth's upper atmosphere and ways to mitigate disturbing effects. Temporal and spatial changes in the upper atmosphere act to limit and degrade the performance of terrestrial and Earth-space radio systems in many different ways and this is why mitigation activities must involve several topics like ionospheric monitoring and modeling, development of new hardware for communication systems and new propagation simulator, measurements and modeling of ionospheric Total Electron Content (TEC) and ionospheric scintillations, using in particular the Global Positioning System (GPS). The European ionospheric community has long been aware that cooperation research on an international basis is essential to deal with such complex issues. In particular, international cooperation is required for the collection of data, in both the real-time and in retrospective modes, the development and verification of

Mailing address: Dr. Alain Bourdillon, IETR, Université de Rennes 1, 35042 Rennes, France; e-mail: alain.bourdillon@univ-rennes1.fr new methods to improve the performance of both operational and future terrestrial and Earth-space communication systems and the exchange of expertise on space plasma effects on Global Navigation Satellite Systems (GNSS).

In this context the COST 296 Action MIERS on the «Mitigation of Ionospheric Effects on Radio Systems» has made a significant impact in a number of areas.

\section{The major achievements of COST 296}

a) A study dedicated to the improvement of the NeQuick model for Galileo users has been performed to identify the weaknesses of the model at mid-latitudes.

b) Neuro-fuzzy modelling based methods to predict foF 2 1-24 hours in advance have been presented.

c) An analytical model has been developed for mid-scale ionospheric inhomogeneities occurring either as patches in the high-latitude ionosphere or bubbles in the low latitude/equatorial ionosphere in order to investigate and predict their scintillation effects.

d) The day-to-day variability in TEC is greater than in $f o F 2$ and exhibits its higher values during the nighttime hours. The slab thickness variability shows a similar pattern with that of TEC or of $f o F 2$ variability.

e) The Prompt Ionospheric Database has been improved (http://www.wdc.rl.ac.uk/cgi- 
bin/digisondes/cost_database.pl). This is 24/7 on-line service that is based on the contributions of a number of COST 296 participating institutions.

f) The RWC-Warsaw/IDCE web service (http://www.cbk.waw.pl/rwc/idce and ftp:// www.cbk.waw.pl/idce) provides on line access to the 24-hours ahead forecast of foF2 and $M(3000) F 2$ for quiet as well as disturbed conditions based on data of continuous monitoring of regional ionospheric conditions from all available sites. The list of available forecasting models.

g) The structure of a heterogeneous array has been proposed for HF direction finding purpose with advantages resulting from its sensibility to the incident polarization.

h) Research has been directed at exploiting MIMO techniques for long-range communications within the HF band. Several HF-MIMO measurement campaigns were performed using different antenna array configurations at both the transmitter and receiver ends of the link.

i) Time-of-flight measurements (TOF) over the radio link between Uppsala (Sweden) and Bruntingthorpe (U.K.) have been performed and compared with the TOF provided by the long-term prediction models SIRM.

1) A long-term interference prediction model for the HF spectrum is being developed. This model could be used in the absence of system capability to assess interference background in real time or near real time.

m) An operational service for space-plasma and space-weather monitoring has been developed (Space Weather Applications Center Ionosphere, http://w3swaci.dlr.de/).

n) The role of higher order ionosphere errors (the term in f-3 due to the effect of the geomagnetic field; the three f-4 terms (i) due to the curvature of the path in the plane of incidence, (ii) curvature perpendicular to the plane of incidence and (iii) due to the actual distribution of electron density along the path) is under consideration.

o) The monitoring of ionospheric scintillation activity has been continued in different parts of the world. The database has been analyzed and the development of forecasting models has been initiated

\section{Proposal for a follow-on Action}

Since 1991, the COST 238, COST 251, COST 271 and COST 296 Actions relating to ionospheric radio propagation have been particularly useful in creating a critical mass of researchers in projects where the initial emphasis was on long-term ionospheric prediction and retrospective ionospheric modeling over Europe.

The COST 238 Action PRIME (Prediction and Retrospective Ionospheric Modeling over Europe) was a four year research project with objectives to develop regional maps and models over the European ionosphere that are more accurate than internationally available global maps and models. It involved the participation of some 72 scientists and engineers from 17 countries. The work encompassed the production of regional monthly median maps and algorithms for generating «instantaneous» snapshot maps of the standard vertical-incidence ionospheric characteristics from coordinated sounding measurements within Europe. Additional studies involved height profiles of electron density, the vertical total electron content up to an altitude of $1000 \mathrm{~km}$ and research leading to an improved understanding and thus potential for better modeling of ionospheric storm morphologies. Work conducted within COST 238 was continued in the follow-on project COST 251 IITS (Improved quality of service in Ionospheric Telecommunication Systems planning and Operation) on the application of PRIME results in the improved quality of service in ionospheric telecommunication systems planning and operation.

Both projects yielded significant results, but they also identified further areas where future development was needed. The topics for more research included: hour-to-hour and day-to-day variability prediction capabilities in the topside ionosphere, upgrading of the current models to include scintillation effects, the prediction of the ionospheric and plasmaspheric effects on navigational systems and the development of methods for calculating the reliability and compatibility of HF radio systems using digital modulation techniques and over the horizon HF backscatter radars. 
The COST 271 Action «Effects of the upper atmosphere on terrestrial and Earth-space communications» was then established to examine a range of relevant research areas within four broad groupings by considering the impact of variability of space environment on communications, to assess space plasma effects for satellite applications, to consider the ionospheric effects on terrestrial communications and the space plasma effects on Earth-space and satellite-to-satellite communications.

The COST 296 Action MIERS is now finishing and it is clear that with the development of GPS applications, the coming Galileo system and the more frequent use of HF communication systems with commercial aircraft, on polar routes for instance, there is a strong requirement for improved understanding of ionospher- ic effects on such systems and better modeling of the ionosphere itself. This is the reason why the ionospheric COST community envisages proposing a new Action.

\section{Conclusion}

The COST 296 Action has been very successful in bringing together in collaborative studies researchers who represent most of the available European expertise on the effects and mitigation of ionospheric effects on radio systems. The significant results and advances in knowledge obtained during COST 296 have been summarized here. In addition a new proposal is currently under consideration. 